\title{
SYMMETRY OF GENERALIZED GROUP ALGEBRAS
}

\author{
KJELD B. LAURSEN
}

In this note we shall consider the generalized group algebras $B^{p}(G, A)$, where $G$ is a compact Hausdorff group, $A$ a Banach-* algebra, and $1 \leqq p<\infty$. These spaces have been studied by Spicer [6] and [7] and are defined as the spaces of functions $f: G \rightarrow A$ for which

$$
\left[\int|f(g)|^{p} d m(g)\right]^{1 / p}<\infty .
$$

$B^{p}(G, A)$ is normed by defining

$$
|f|_{p}=\left[\int|f(g)|^{p} d m(g)\right]^{1 / p}
$$

and involution is defined as usual: $f^{*}(g)=f\left(g^{-1}\right)^{*}$.

We prove the following

THEOREM. If $G$ is a compact group and $A$ is a Banach algebra with (continuous) involution, then $B^{p}(G, A)$ is symmetric if and only if $A$ is symmetric.

A Banach- * -algebra is symmetric if elements $f^{*} * f$ have nonnegative spectrum. This is the case if and only if hermitian elements have real spectra [5].

We first observe that it suffices to show that $A$ is symmetric iff $B^{1}(G, A)$ is symmetric, because $B^{1}(G, A)$ symmetric $\Rightarrow B^{p}(G, A)$ symmetric for any $p, 1 \leqq p<\infty, \Rightarrow A$ symmetric $\Rightarrow B^{1}(G, A)$ symmetric. The proof of the first of these implications will be accomplished by showing that if $t \in B^{p}(G, A) \subseteq B^{1}(G, A)$ then the spectrum of $t$ in $B^{p}(G, A), \sigma_{p}(t)$ equals the spectrum of $t$ in $B^{1}(G, A), \sigma_{1}(t)$. Since $B^{p}(G, A) \subseteq B^{1}(G, A)$, clearly $\sigma_{1}(t) \subseteq \sigma_{p}(t)$. On the other hand, $B^{p}(G, A)$ is an ideal in $B^{1}(G, A)$ [6]. Recall that $0 \neq \lambda \notin \sigma_{1}(t)$ iff $t / \lambda$ has a quasiinverse, $y_{\lambda}$, say, in $B^{1}(G, A)\left[4\right.$, p. 28]. $t / \lambda$ and $y_{\lambda}$ satisfy the relationship

$$
t / \lambda+y_{\lambda}-(t / \lambda) * y_{\lambda}=0
$$

or

$$
y_{\lambda}=(t / \lambda) * y_{\lambda}-t / \lambda .
$$

Using the fact that $B^{p}(G, A)$ is an ideal in $B^{1}(G, A)$ we conclude

Received by the editors June 4, 1969. 
that $y_{\lambda} \in B^{p}(G, A)$ i.e. $\lambda \notin \sigma_{p}(t)$. If neither $B^{p}(G, A)$ nor $B^{1}(G, A)$ contains an identity $0 \in \sigma_{1}(t)$ and $0 \in \sigma_{p}(t)$. On the other hand, if $G$ is a finite group, then obviously $t \in B^{p}(G, A)$ has an inverse in $B^{p}(G, A)$ if and only if $t$ has an inverse in $B^{1}(G, A)$, because $B^{1}(G, A)=B^{p}(G, A)$ setwise. Consequently, we have shown

Lemma 1. $\sigma_{1}(t)=\sigma_{p}(t)$ for all $t \in B^{p}(G, A)$ and any $p, 1 \leqq p<\infty$.

From this lemma it follows that if $B^{1}(G, A)$ is symmetric and $t \in B^{p}(G, A)$, then $-1 \notin \sigma_{1}\left(t^{*} t\right)$ and therefore $-1 \notin \sigma_{p}\left(t^{*} t\right)$. This shows that $-t^{*} t$ is quasi-regular in $B^{p}(G, A)$ for any $t \in B^{p}(G, A)$; therefore $B^{p}(G, A)$ is symmetric if $B^{1}(G, A)$ is symmetric.

Next we prove the second implication. Suppose $B^{p}(G, A)$ is symmetric. We show that $A$ is symmetric: Simply embed $A$ in $B^{p}(G, A)$ by considering the isometric image of $A$ in $B^{p}(G, A)$. This identification shows immediately that if $B^{p}(G, A)$ is symmetric, then $A$ is symmetric.

To show that $B^{1}(G, A)$ is symmetric, provided that $A$ is, is somewhat more complicated. The proof given here depends on the minimal ideal structure of $L^{1}(G)$ via the identification $B^{1}(G, A)=L^{1}(G) \otimes_{\gamma} A$ [6], based on a result by Grothendieck [1, p. 59]. We present the proof as a sequence of lemmas.

The first two of these are proved in [7].

Lemma 2. Let $X_{1}$ be a finite-dimensional Banach space and $X_{2}$ be any Banach space. Let $\left\{l_{1}, \cdots, l_{n}\right\}$ be any basis of unit vectors for $X_{1}$; if $t \in X_{1} \otimes X_{2}$ then $t$ has a unique representative $t=\sum_{i=1}^{n} l_{i} \otimes y_{i}$. Define

$$
\phi: X_{1} \otimes X_{2} \rightarrow \sum \oplus_{n} X_{2} \quad \text { by } \quad \phi(t)=\left(y_{1}, \cdots, y_{n}\right) .
$$

$\phi$ is an algebraic isomorphism onto.

LeMma 3. $X_{1}$ and $X_{2}$ as in Lemma 2. If we norm $\sum \oplus_{n} X_{2}$ by defining

$$
\left|\left(y_{1}, \cdots, y_{n}\right)\right|=\sum_{i=1}^{n}\left|y_{i}\right| \text {, }
$$

then $\phi$ as defined above is a homeomorphism of $\sum \oplus_{n} X_{2}$ and $X_{1} \otimes_{\gamma} X_{2}$.

Note that since $X_{1}$ is finite-dimensional the algebraic tensor product normed with the greatest cross norm is complete.

Lemma 4. Suppose $X_{1}$ is a simple finite-dimensional annihilator algebra with proper involution (see [4]) and continuous quasi-inversion. Suppose $X_{2}$ is a Banach-*-algebra. Then $\phi$ defined in Lemma 2 is a *-algebra-isomorphism. 
Proof. The assumptions on $X_{1}$ are made simply to ensure that $X_{1}$ has a basis $\left\{e_{i}\right\}$ consisting of orthonormal, hermitian idempotents $[3$, p. 330$]$, i.e. $\left\{e_{i}\right\}$ satisfies

$$
e_{i} e_{j}=\delta_{i j} e_{j}, \quad \text { for all } i, j=1, \cdots, n,
$$

and

$$
e_{i}^{*}=e_{i}, \quad \text { for all } i=1, \cdots, n .
$$

If $t \in X_{1} \otimes_{\gamma} X_{2}$ then as before $t=\sum e_{i} \otimes y_{i}$ and $\phi(t)=\left(y_{1}, \cdots, y_{n}\right)$. Hence $t^{*}=\sum e_{i}^{*} \otimes y_{i}^{*}=\sum e_{i} \otimes y_{i}^{*}$ and $\phi\left(t^{*}\right)=\left(y_{1}^{*}, \cdots, y_{n}^{*}\right)=[\phi(t)]^{*}$. Moreover, if $t_{1}=\sum e_{i} \otimes x_{i}$ and $t_{2}=\sum e_{j} \otimes y_{j}$ then

so that

$$
t_{1} t_{2}=\sum_{i j} e_{i} e_{j} \otimes x_{i} y_{j}=\sum_{i} e_{i} \otimes x_{i} y_{i}
$$

$$
\begin{aligned}
\phi\left(t_{1} t_{2}\right) & =\left(x_{1} y_{1}, \cdots, x_{n} y_{n}\right)=\left(x_{1}, \cdots, x_{n}\right)\left(y_{1}, \cdots, y_{n}\right) \\
& =\phi\left(t_{1}\right) \phi\left(t_{1}\right) .
\end{aligned}
$$

Corollary 1. $X_{1}$ and $X_{2}$ as in Lemma 4. $X_{1} \otimes_{\gamma} X_{2}$ is symmetric if and only if $X_{2}$ is symmetric.

Remark. The assumptions about $X_{1}$ imply that $X_{1}$ is symmetric $[4$, p. 266].

Proof. By Lemma 3 and Lemma 4 it suffices to show that $\sum_{n} \oplus X_{2}$ is symmetric iff $X_{2}$ is symmetric. But this is an immediate consequence of the fact that

$$
\sigma\left(y_{1}, \cdots, y_{n}\right)=\bigcup_{i=1}^{n} \sigma\left(y_{i}\right)
$$

for any $\left(y_{1}, \cdots, y_{n}\right) \in \sum \oplus_{n} X_{2}$.

We now specialize to $B^{1}(G, A)=L^{1}(G) \otimes_{\gamma} A$. The theory to be developed depends on the minimal ideal structure of $L^{1}(G)$.

If $S \subset L^{1}(G)$ then $(S \otimes A)_{\gamma}$ will denote the $\gamma$-closure of $S \otimes A$ in $L^{1}(G) \otimes_{\gamma} A$.

Lemma 5. If $M \subset L^{1}(G)$ is a minimal two-sided closed ideal, then $(M \otimes A)_{\gamma}$ is a closed ideal in $B^{1}(G, A)$, symmetric if and only if $A$ is symmetric.

Proof. Since $G$ is compact, $M$ is a finite-dimensional simple annihilator algebra with proper involution and continuous quasi-inversion $[3, \mathrm{VI}]$. Clearly $(M \otimes A)_{\gamma}$ is a closed ideal; if $t_{1}=\sum_{i=1}^{\infty} x_{i} \otimes y_{i} \in M \otimes A$ and 


$$
t_{2}=\sum_{j=1}^{\infty} u_{j} \otimes v_{j} \in L^{1}(G) \otimes_{\gamma} A
$$

then

$$
t_{1} t_{2}=\sum_{i, j} x_{i} u_{j} \otimes y_{i} v_{j} \in(M \otimes A)_{\gamma} .
$$

Similarly $t_{2} t_{1} \in(M \otimes A)_{\gamma}$. It is easy to see that $M \otimes_{\gamma} A$ and $(M \otimes A)_{\gamma}$ are ${ }^{*}$-isomorphic, using the technique of Lemma 2. Since $M \otimes_{\gamma} A$ is symmetric iff $A$ is symmetric, the conclusion follows by the above and Lemma 4.

Lemma 6. Let $\left\{M_{i}\right\}_{i=1}^{n}$ be minimal two-sided ideals of $L^{1}(G)$; if $A$ is symmetric, then $\sum_{i=1}^{n} \oplus\left(M_{i} \otimes A\right)_{\gamma} \subset B^{1}(G, A)$ is symmetric.

Proof. If $A$ is symmetric, then $\left(M_{i} \otimes A\right)_{\gamma}$ is symmetric (Lemma 5). The rest follows as in the proof of Corollary 1.

Now we are able to complete the proof of the theorem. Suppose $A$ is symmetric. We must show that $B^{1}(G, A)$ is symmetric; from this it will follow that $B^{p}(G, A)$ is symmetric (Lemma 1$)$. In accordance with [5] we show that hermitian elements in $B^{1}(G, A)$ have real spectra. Adapting a construction in $[2$, Theorem (28.53)] to the present situation we can find a net of functions $\left\{u_{\alpha}\right\}$ with the following properties:

(i) each $u_{\alpha}$ is complex-valued continuous, nonnegative, positivedefinite and central.

(ii) $\int u_{\alpha} d m=1$ for all $\alpha$.

(iii) $u_{\alpha} * f=f * u_{\alpha} \rightarrow f$ for any $f \in L^{1}(G)$.

Clearly each $u_{\alpha}$ generates an operator $T_{\alpha}$ in $B^{1}(G, A)$ defined as follows

$$
\begin{aligned}
f & =\sum x_{i} \otimes y_{i} \in B^{1}(G, A)=L^{1}(G) \otimes_{\gamma} A \Rightarrow \\
T_{\alpha} f & =\sum u_{\alpha} * x_{i} \otimes y_{i} .
\end{aligned}
$$

Equally clear is it that $T_{\alpha}$ approximates the identity of $B^{1}(G, A)$, i.e.

$$
\sum u_{\alpha} * x_{i} \otimes y_{i} \rightarrow \sum x_{i} \otimes y_{i}
$$

Now, let $f$ be a hermitian element of $B^{1}(G, A)$. We will use the notation $u_{\alpha} * f$ for $T_{\alpha} f \in B^{1}(G, A)$. From $\left\{u_{\alpha} * f\right\}$ we can pick a sequence $\left\{u_{n} * f\right\}$ such that

$$
\left|u_{n} * f-f\right|<1 / n, \quad n=1,2, \cdots .
$$

Let $\mathfrak{F}=\{M\}$ be the collection of minimal two-sided ideals in $L^{1}(G)$. Since $G$ is compact any irreducible representation of $L^{1}(G)$ is 
realizable as left translation in some $M \in \mathcal{F}[3$, p. 434]. Moreover, each $u_{n}$ being positive definite we have that

$$
u_{n}(\cdot)=\sum_{M \in \mathcal{F}} c_{n}(M) \chi_{M}(\cdot)
$$

where $\chi_{M}$ is the character of $M$, where $c_{n}(M) \geqq 0$ and where $\sum_{M \in \mathcal{F}} c_{n}(M) \chi_{M}(e)<\infty$. Again following [2], for each $u_{n}$ we can choose a finite partial sum, $u_{n}^{\prime}$, of the series for $u_{n}$ so that $\left|u_{n}^{\prime}-u_{n}\right|_{\infty}$ $<1 / 2 n$. Setting $u_{n}^{\prime \prime}=\frac{1}{2}\left(u_{n}^{\prime}+u_{n}^{\prime *}\right)$ we get a continuous hermitian central trigonometric polynomial for which $\left|u_{n}^{\prime \prime}-u_{n}\right|_{\infty}<1 / n$. If we normalize $u_{n}^{\prime \prime}$ to obtain $v_{n}$, i.e.

$$
v_{n}=u_{n}^{\prime \prime} /\left|u_{n}^{\prime \prime}\right|_{1}, \quad n=1, \cdots,
$$

then it is clear that $v_{n} * f \rightarrow f$. Since $v_{n} * f=f * v_{n}$ each $v_{n} * f$ is hermitian. Also since $v_{n} * f$ and $f$ commute we can make use of $[4,(1.6 .17)]$. Consequently, it suffices to show that $v_{n} * f$ has a real spectrum. But since $v_{n}$ is a finite linear combination of characters it follows that

$$
v_{n} * f \in \sum_{i \in K} \oplus\left(M_{i} \otimes A\right)_{\gamma}
$$

where $K$ is finite set. Lemma 6 then implies that $v_{n} * f$ has real spectrum. This completes the proof.

Corollary. Let $G$ be a compact group and $H$ a locally compact group. $L^{1}(G \times H)$ is symmetric if and only if $L^{1}(H)$ is symmetric.

\section{BIBLIOGRAPHY}

1. A. Grothendieck, Produits tensoriels topologiques et espaces nucléaires, Mem. Amer. Math. Soc. No. 16 (1955). MR 17, 763.

2. E. Hewitt and K. Ross, Abstract harmonic analysis. Vol. II, Die Grundlehren der math. Wissenschaften, Band 152, Springer-Verlag, Berlin, and New York, 1970.

3. M. NaǏmark, Normed rings, GITTL, Moscow, 1956; English transl., Noordhoff, Groningen, 1959; rev. ed., 1964. MR 19, 870; MR 22 \#1824; MR 34 \#4928.

4. C. E. Rickart, General theory of Banach algebras, The University Series in Higher Mathematics, Van Nostrand, Princeton, N. J., 1960. MR 22 \#5903.

5. S. Shirali and J. W. M. Ford, Symmetry in complex involutory Banach algebras. II, Duke Math. J. (to appear).

6. D. Spicer, Group algebras of vector-valued functions, Pacific J. Math. 24 (1968), 379-399. MR 37 \#2001.

7. - Semisimplicity of group algebras of vector-valued functions, Proc. Amer. Math. Soc. 19 (1968), 573-577. MR 37 \#2002.

University of Aarhus, Aarhus, Denmark 